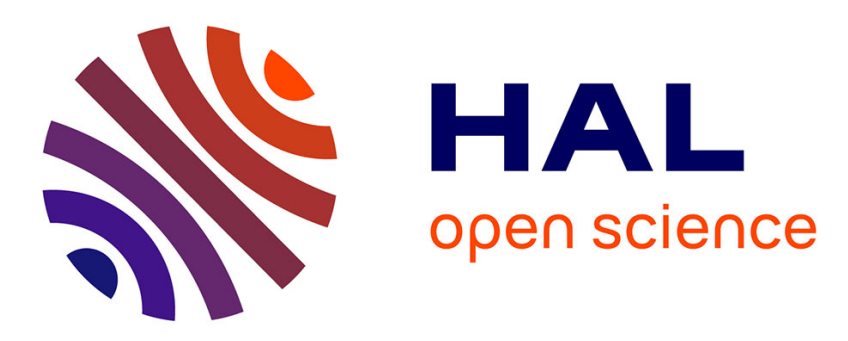

\title{
Picosecond and nanosecond polychromatic pump-probe studies of bubble growth in carbon-nanotube suspensions
}

L. Vivien, D. Riehl, J.-F. Delouis, J. Delaire, François Hache, E. Anglaret

\section{- To cite this version:}

L. Vivien, D. Riehl, J.-F. Delouis, J. Delaire, François Hache, et al.. Picosecond and nanosecond polychromatic pump-probe studies of bubble growth in carbon-nanotube suspensions. Journal of the Optical Society of America B, 2002, 19 (2), pp.208-214. 10.1364/JOSAB.19.000208 . hal-00836939

HAL Id: hal-00836939

https://hal-polytechnique.archives-ouvertes.fr/hal-00836939

Submitted on 5 May 2014

HAL is a multi-disciplinary open access archive for the deposit and dissemination of scientific research documents, whether they are published or not. The documents may come from teaching and research institutions in France or abroad, or from public or private research centers.
L'archive ouverte pluridisciplinaire HAL, est destinée au dépôt et à la diffusion de documents scientifiques de niveau recherche, publiés ou non, émanant des établissements d'enseignement et de recherche français ou étrangers, des laboratoires publics ou privés. 


\title{
Picosecond and nanosecond polychromatic pump-probe studies of bubble growth in carbon-nanotube suspensions
}

\author{
Laurent Vivien and Didier Riehl \\ Délégation Générale pour l'Armement/Centre Technique d'Arcueil/Department Laser, Optique et Thermo-optique, \\ Arcueil, France
}

Jean-François Delouis and Jacques A. Delaire

Laboratoire de Photophysique et Photochimie Supramoléculaires et Macromoléculaires, Unité Mixte de Recherche, Centre National de la Recherche Scientifique 8531, ENS Cachan, France

François Hache

Ecole Polytechnique, Laboratoire d'Optique et Biosciences, Unité Mixte de Recherche, Centre National de la Recherche Scientifique 7645, Palaiseau, France

\section{Eric Anglaret}

Université de Montpellier II, Groupe de Dynamique des Phases Condensées, Unité Mixte de Recherche, Centre National de la Recherche Scientifique 5581, Montpellier, France

Received February 27, 2001; revised manuscript received July 25, 2001

\begin{abstract}
Optical limiting in carbon-nanotube suspensions, whose origin lies in a strong nonlinear scattering due to solvent vapor bubbles and sublimation of the nanotubes, is investigated in the picosecond and nanosecond regimes by polychromatic pump-probe experiments. Samples were pumped either with 532-nm or 1064-nm pulses, and probed from $400 \mathrm{~nm}$ to $650 \mathrm{~nm}$. Using a model based on Mie theory, we determine the time evolution of the radius and the concentration of the scattering centers for both temporal regimes. We compare the transmission signals for single-wall carbon nanotubes suspended in water and in chloroform and for multiwall carbon nanotubes in water. Several conclusions can be drawn. First, coalescence of gaseous cavities is more effective in water than in chloroform, leading to nonlinear scattering by a smaller number of larger bubbles. Second, in spite of the smaller size of the scattering centers, the limiting efficiency of chloroform suspensions is better than that of water suspensions, due to a larger volume fraction of the gaseous phase. However, the characteristic times for the growth of laser-induced bubbles are too long to allow efficient limiting of subnanosecond laser pulses. (C) 2002 Optical Society of America
\end{abstract}

OCIS codes: $190.3970,190.4400,190.4870,290.4020,290.5850$.

\section{INTRODUCTION}

There is considerable interest in the application of the nonlinear optical properties of materials to optical limiting. ${ }^{1,2}$ Indeed, the proliferation of laser-based systems is associated with potential harmful effects from these bright, coherent light sources. Nowadays, laser sources (optical parametric oscillators, dye lasers, Raman lasers, ...) are widely used in many applications, not only in the laboratory, but also in many areas of industry and medicine as well as for military applications, and they constitute a potential hazard for eyes and other optical sensors (CCD, thermal camera, ...). It is therefore crucial to protect all sensors against this threat by use of optical limiters. The ideal optical limiter would have high broadband linear transmittance for low input fluences, and the output energy must always remain below the damage threshold of sensors (e.g., camera or human eyes). In addition, this ideal limiter should be efficient from a few picoseconds up to longer pulse durations (from nanosecond to millisecond) on a broad range of wavelengths (visible and near infrared). Numerous mechanisms have been proposed for optical-limiting applications. Among them, reverse saturable absorption ${ }^{3}$ has demonstrated a good efficiency but mainly on relatively narrow spectral bands. Broadband optical limiting and colorimetric neutrality can be obtained by associating several narrow-band reverse saturable absorption molecules, but such a material generally exhibits a strongly reduced linear transmittance. ${ }^{4}$ On the other hand, multiphoton absorbers exhibit an excellent transparency and possess broadband-limiting properties, but only for short laser pulses $(\tau \leqslant 10 \mathrm{~ns})$. In contrast, nonlinear-refractive $e^{5,6}$ and nonlinear-scattering ${ }^{7-10}$ materials, as, for example, carbon-black suspensions, ${ }^{7-9}$ can also provide broadband optical limiting, with better performances for longer laser pulses. Following the discovery of carbon nanotubes by Iijima in 1991, ${ }^{11}$ many research efforts were focused on this new form of carbon to clarify its attractive physical 
properties and in particular nonlinear optical effects. Since 1998, optical-limiting properties of carbon nanotubes have been the object of numerous investigations, revealing that these materials are promising candidates for optical limiting in the visible and near-infrared domains. $^{12-19}$ They present a broadband transparency at low input fluences (from 400 to $1100 \mathrm{~nm}$ ), with excellent colorimetric neutrality. The origin of optical limiting in the nanosecond regime is a strong nonlinear scattering induced by the sublimation of the laser-irradiated carbon nanotubes and by the formation of solvent bubbles due to heat transfer from the particles to the surrounding liquid. ${ }^{16-18}$

In this study, we report picosecond and nanosecond pump-probe results for three kinds of samples: singlewall carbon nanotubes (SWNT) suspended in water and in chloroform and multiwall carbon nanotubes (MWNT) suspended in water, with 532-nm and 1064-nm pump pulses and a polychromatic probe beam that allows determination of the kinetic of bubble formation and coalescence.

Section 2 presents the synthesis method and the purification procedure of single-wall and multiwall carbon nanotubes. Section 3 reports the results obtained with 30-ps pump pulses, with a comparison between SWNT and MWNT at different pump fluences and probe wavelengths. In the same section, we also report on nanosecond pump-probe experiments. In Section 4 we present a simple calculation that allows estimation of the number and the average radius of the scattering centers responsible for the observed drop of probe transmittance. We thus obtain the evolution of the volume fraction of the gas cavities that scatters the probe beam, and we observe coalescence and condensation effects.

\section{MATERIALS}

Carbon nanotubes were synthesized by the electric arc discharge technique. ${ }^{20}$ An electric arc discharge is created between two graphite electrodes under a helium atmosphere in the presence of a metallic catalyst ( $\mathrm{Ni}$ and $\mathrm{Y}$ with 4.2:1 at.\%) for SWNT synthesis and with only pure graphite electrodes for MWNT. As-prepared samples contain impurities such as amorphous carbon, graphite, fullerenes, and residual catalysts. The SWNT samples were purified in a three-step procedure. ${ }^{21}$ The first step consists of a nitric acid treatment at $100^{\circ} \mathrm{C}$, which partially disintegrates the catalyst particles and disentangles the complex network of nanotubes, other carbonous materials, and catalyst particles. The second one is a tangential filtration that allows separation of the nanotubes from the other species. Finally, a thermal treatment under inert atmosphere eliminates residual catalysts. After such a purification procedure, the amount of SWNT is close to 90 vol. $\%$ in the samples. SWNT are observed to usually self-assemble on a triangular array into crystalline nanobundles of a couple to some tens of tubes. $^{20,22}$ The diameter of the tubes lies between 1.3 and $1.5 \mathrm{~nm},{ }^{20,23}$ and their length is about several micrometers. For MWNT, amorphous carbon and fullerenes were eliminated by a thermal treatment at $600{ }^{\circ} \mathrm{C}$. The amount of MWNT is close to $80 \mathrm{vol} . \%$, and their external diameter ranges from 2 to $20 \mathrm{~nm}$ depending on the number of layers. SWNT were dispersed in chloroform without surfactant and in water with surfactant (triton X100). MWNT were only dispersed in water/ surfactant. For pump-probe experiments, we studied our suspensions in 1-mm-thick cells, coupled with a circulation system. The linear transmittances of the colorless samples were larger than $80 \%$ from 400 to $1100 \mathrm{~nm}$, and all samples were adjusted at the same linear transmittance.

\section{PUMP-PROBE EXPERIMENTS}

\section{A. Picosecond Studies}

The experimental setup is shown on Fig. 1. We use a mode-locked Nd:YAG laser delivering 30-ps pulses either at $532 \mathrm{~nm}$ or at $1064 \mathrm{~nm}$, at a repetition rate of $8 \mathrm{~Hz}$. The beam is split into two parts; the first one, focused into the nanotube suspensions by use of a $150-\mathrm{mm}$ focallength lens, is used as a pump beam. After passing through the sample, the transmitted pump beam is blocked by a dump. The polychromatic probe beam is obtained by focusing the second Nd:YAG beam on a tungsten electrode under xenon atmosphere (pressure 2 bars), leading to a broadband emission (from $350 \mathrm{~nm}$ to $700 \mathrm{~nm}$ ) with a typical lifetime of $50 \mathrm{~ns}$ (rise time of $\sim 30 \mathrm{ps}$ ). The resulting emitted light is collimated by a short-focallength lens and focused into carbon-nanotube cells, nearly collinear to the pump beam, the probe diameter in the cell being always smaller than the pump-beam diameter $(\sim 450 \mu \mathrm{m})$. The probe transmittance is recorded with a monochromator coupled with a streak camera (ARP, Strasbourg, France, resolution of $8 \mathrm{ps)} \mathrm{in} \mathrm{single-shot}$ mode. A retardation line on the pump path ensures that the sample is probed before and after perturbation. Such a technique allows both spectrally and temporally resolved measurements to be obtained. In order to avoid cumulative thermal effects, we use a circulation system in the cells to refresh nanotube suspensions between consecutive shots. The signal-to-noise ratio is enhanced by averaging 100 consecutive measurements.

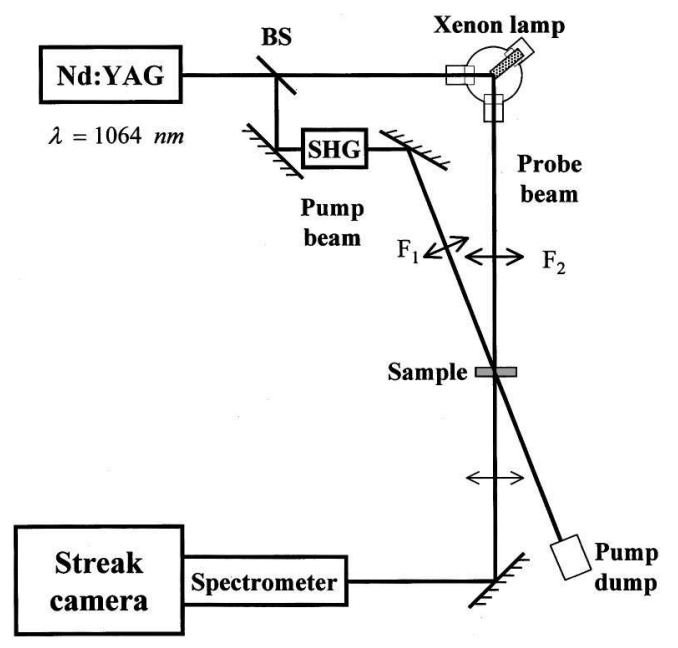

Fig. 1. Pump-probe experimental setup for pump wavelengths 532 and $1064 \mathrm{~nm}$ and probe wavelengths from $400 \mathrm{~nm}$ to $700 \mathrm{~nm}$. BS, beam splitter; F1 and F2, 150-mm and 100-mm focal length, respectively. 


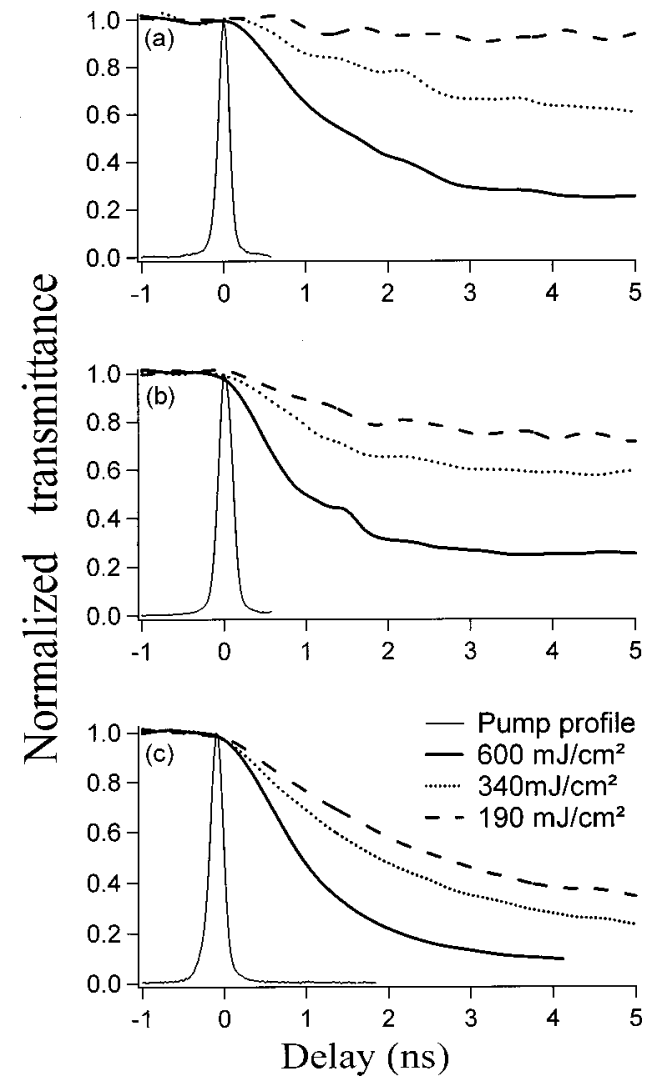

Fig. 2. Probe transmittance, normalized to the linear transmittance as function of delay between the probe at $480 \mathrm{~nm}$ and the pump at $532 \mathrm{~nm}$ for different picosecond pump fluences (from $190 \mathrm{~mJ} / \mathrm{cm}^{2}$ to $600 \mathrm{~mJ} / \mathrm{cm}^{2}$ ) for (a) MWNT in water, SWNT in (b) water, and in (c) chloroform. The linear transmittance is $80 \%$ for all samples.

Figure 2 displays the evolution of probe transmittance at three different pump fluences $\left(190 \mathrm{~mJ} / \mathrm{cm}^{2}, 350\right.$ $\mathrm{mJ} / \mathrm{cm}^{2}$, and $630 \mathrm{~mJ} / \mathrm{cm}^{2}$ ), for SWNT suspended in both water and chloroform solvents and for MWNT suspended in water. The pump wavelength is $532 \mathrm{~nm}$ and the samples are probed at $480 \mathrm{~nm}$, sufficiently far from the pump wavelength to allow an efficient filtering of scattered pump light. The nonlinear response of the samples is not instantaneous, as expected from the probable origin of optical-limiting behavior of carbon nanotubes. In fact, the perturbation begins a few hundreds of picoseconds after the pump pulse; then the minimum probe transmittance is reached in a few nanoseconds (typically $3-5 \mathrm{~ns}$, depending on pump fluence). As the pump fluence increases, the perturbation occurs earlier after the pump pulse and develops faster; however, no limiting of 30-ps pulses occurs. Such a behavior is consistent with previously published picosecond and nanosecond pump-probe results ${ }^{15,16}$ and is in accordance with the proposed mechanism of nonlinear scattering: scattering centers are generated both by solvent-bubble growth and by phase change (sublimation) of the carbon nanotubes, due to intense heating of the particles and subsequent heat transfer. The characteristic times for the growth of the scattering centers are in the nanosecond range.

Let us compare the different behaviors of the three kinds of samples (Fig. 2). In the same solvent (water), SWNT and MWNT exhibit similar responses, but with faster growth of the scattering centers in the case of SWNT. The delay between the pump pulse and the beginning of the probe-transmittance decrease is shorter for SWNT than for MWNT at low pump fluences. This may be related to the smaller external specific area of MWNT as compared with SWNT, which may slow down thermaltransfer processes. Results obtained with SWNT suspended in both liquids (water and chloroform) show a noticeable solvent effect, with a decrease of the probe transmittance significantly larger for the chloroform suspension than for the water sample. It has been shown ${ }^{7,16}$ that the thermodynamic properties of chloroform favor solvent-bubble growth, leading to larger scattering centers.

In order to better understand the nonlinear phenomena in carbon nanotube suspensions, we studied the wavelength dependence of the probe perturbation at a fixed pump wavelength (532 nm or $1064 \mathrm{~nm}$ ). The temporal shapes of the curves obtained for different pump fluences are similar to those in Fig. 2, but the magnitude of the drop of the transmittance is strongly wavelength dependent. Figures 3(a) and 3(b) compare the wavelength dependence of the probe transmittance measured at $4 \mathrm{~ns}$ after the top of the pump pulse, for 532-nm and 1064-nm pump wavelengths (See an example of the total time dependence in Fig. 4). The behavior is the same for the two wavelengths within the experimental errors. We observe

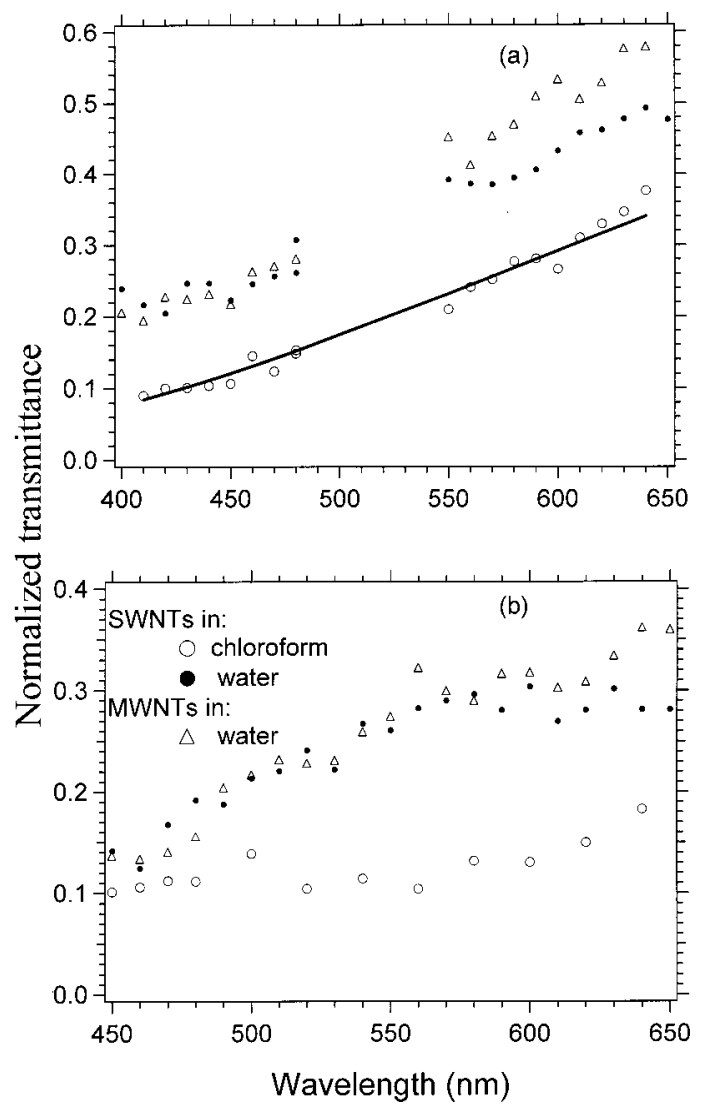

Fig. 3. Normalized probe transmittance as a function of probe wavelength for SWNT in water and chloroform and for MWNT in water, for a pump wavelength of (a) $532 \mathrm{~nm}$ and (b) $1064 \mathrm{~nm}$. The delay between the pump and the probe is $4 \mathrm{~ns}$, and the pump energy is $600 \mathrm{~mJ} / \mathrm{cm}^{2}$. The solid curve is a fit obtained with Mie theory. The linear transmittance is $80 \%$ for all samples. 


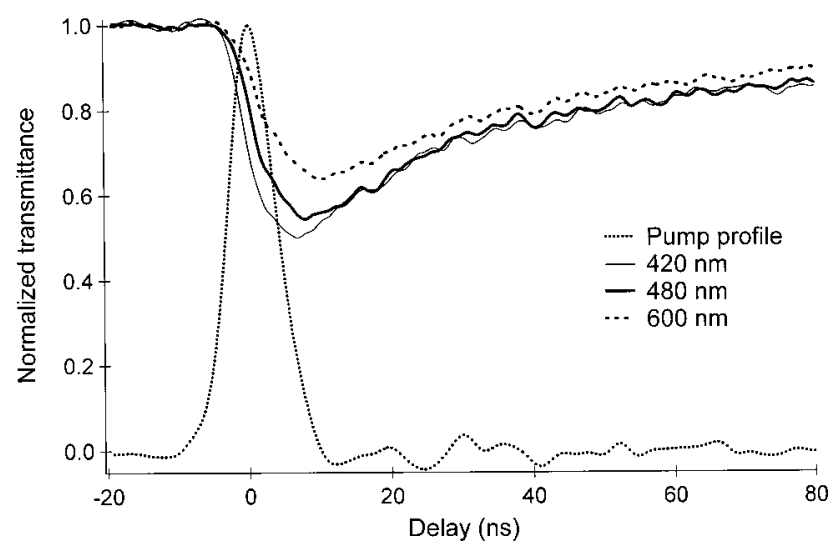

Fig. 4. Probe perturbation at 420,480 , and $600 \mathrm{~nm}$ for SWNT in water at $10 \mathrm{~ns}$ and at $532 \mathrm{~nm}$ for input fluence of $200 \mathrm{~mJ} / \mathrm{cm}^{2}$. The linear transmittance is $80 \%$ for all samples.

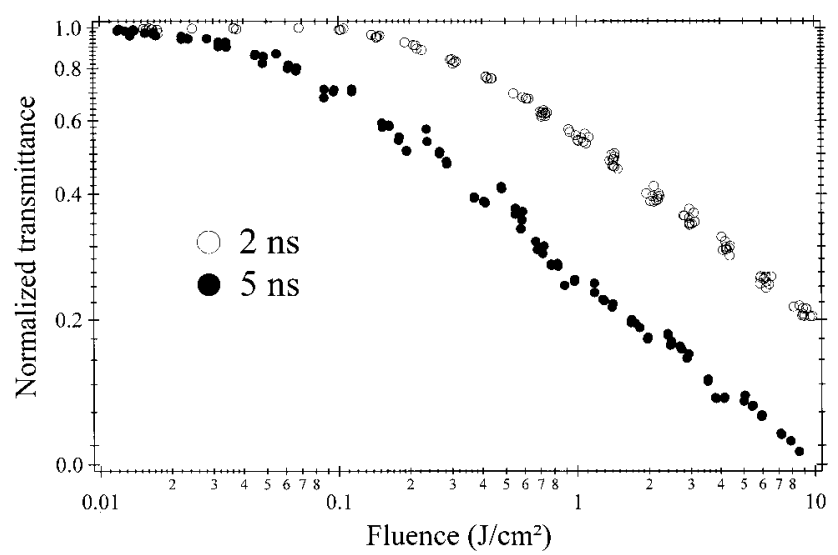

Fig. 5. Optical-limiting curves of SWNT suspended in chloroform for 2- and 5-ns pulse widths at $532 \mathrm{~nm}$. The linear transmittance is $70 \%$ for all samples.

a broadband-limiting efficiency from $400 \mathrm{~nm}$ to $650 \mathrm{~nm}$ for all the samples. As expected, from Mie-scattering theory (solid curve in Fig. 3(a); see Section 4), the shorter the wavelength, the lower the transmittance. Indeed, for submicronic particles, scattering is more efficient when the size of the scattering centers is close to the wavelength, or reciprocally, for given scattering centers, when the wavelength decreases. A quantitative analysis of the wavelength dependence of the transmittance allows determination of the concentration and average radius of the scattering centers. Such an analysis is performed in Section 4.

\section{B. Nanosecond Studies}

In order to better understand the different opticallimiting performances obtained in the nanosecond regime, we carried out nonlinear-transmittance experiments for different pulse durations. The nanosecond data support the results presented in Subsection 3.A. We realized similar pump-probe experiments with a $\mathrm{Nd}$ :YAG laser delivering 10-ns pulses width at $532 \mathrm{~nm}$. As for picosecond pump-probe experiments, we performed transmittance measurements for different probe wavelengths (from $400 \mathrm{~nm}$ to $650 \mathrm{~nm}$ ) and recorded the probe transmittance of the carbon-nanotube samples at several delays (from 1 to $80 \mathrm{~ns}$ ). Figure 4 shows the transmittance kinetics of SWNT suspended in water, for a pump fluence of $200 \mathrm{~mJ} / \mathrm{cm}^{2}$ and for three different probe wavelengths: 420,480 , and $600 \mathrm{~nm}$. The minimum of transmittance is reached at $\sim 7 \mathrm{~ns}$ and $\sim 10 \mathrm{~ns}$ after the top of the pump pulse for SWNT and MWNT (not shown), respectively. As is explained in Section 4, this minimum corresponds to a maximum volume fraction of vapor bubbles, and thus to maximum extinction, followed by a slow relaxation process. These curves are in agreement with our previously published results, ${ }^{17}$ but polychromatic measurements allow performance of a deeper analysis.

Figure 5 shows the limiting curves obtained with the same SWNT/chloroform sample at the same wavelength (532 nm), obtained by two different lasers under similar focusing conditions: a Nd:YAG laser delivering 5-ns FWHM pulses and an optical parametric oscillator delivering 2-ns FWHM pulses. The limiting performances obtained with 5-ns pulses are considerably better than those obtained at $2 \mathrm{~ns}$, with a lower limiting threshold ( $\sim 40$ $\mathrm{mJ} / \mathrm{cm}^{2}$ instead of $150 \mathrm{~mJ} / \mathrm{cm}^{2}$ ) and a transmittance reduced by a factor of 2 for incident fluences $>0.5 \mathrm{~J} / \mathrm{cm}^{2} .18$ The explanation is that in the nanosecond regime, the maximum size of the scattering centers is reached only after the end of the incident pulses, even at $10 \mathrm{~J} / \mathrm{cm}^{2}$. Therefore one observes a strong pulse-duration dependence of the limiting performances. This also explains that no optical limiting at all is observed in the picosecond range.

\section{INTERPRETATION}

In our previous investigations on the optical-limiting origin in carbon nanotube suspensions, ${ }^{16,17}$ we demonstrated a strong nonlinear-scattering effect principally due to the growth of vapor bubbles, which can be solvent bubbles or carbon vapor bubbles, depending on the incident fluence and on the thermodynamic properties of the solvent. In all cases, the threshold for solvent-bubble formation is significantly lower than the carbon sublimation threshold. ${ }^{16,17}$ However, solvent-bubble growth is much slower than expansion of vaporized carbon cavities. ${ }^{16,17}$ For example, with 5-ns pulses, solvent-bubble formation occurs at incident fluences as low as $10 \mathrm{~mJ} / \mathrm{cm}^{2}$ but only a few tens of nanoseconds after the pump pulse and thus does not contribute to optical limiting. ${ }^{17}$ The limiting threshold, $\sim 150 \mathrm{~mJ} / \mathrm{cm}^{2}$ at 1064-nm pump wavelength, corresponds to carbon-nanotube sublimation. On the contrary, solvent-bubble growth contributes very efficiently to optical limiting of 80-ns duration pulses in carbon nanotube/chloroform suspensions. ${ }^{18}$

The analysis of the extinction of the polychromatic probe beam allows estimation of the evolution of size and dimensions of the scattering centers during and after the pump pulse, from Mie theory for both pump temporal regimes (30 ps and $10 \mathrm{~ns}$ ). For this, we developed a simple model, where we considered the following approximations: the bubbles are spherical, their index of refraction is 1 , and they are considered to be monodisperse in size. Moreover, we assume that only simple scattering occurs (we neglect multiple scattering). The assumption of spherical bubbles may be erroneous for carbon nanotubes, 
but, beside the simplification it brings, it is justified by the fact that the nanotubes are entangled in coils when in solution.

The transmittance of the samples follows the BeerLambert law:

$$
T_{N}=\exp \left(-\eta l \sigma_{\text {ext }}\right)
$$

where $T_{N}$ is the transmittance, $\eta$ is the volume concentration of scattering centers $\left(\mathrm{m}^{-3}\right), l$ is the thickness of the cell $(\mathrm{m})$, and $\sigma_{\text {ext }}$ is the extinction cross section $\left(\mathrm{m}^{2}\right)$, which reads

$$
\sigma_{\text {ext }}=\sigma_{\mathrm{abs}}+\sigma_{\mathrm{sc}}
$$

where $\sigma_{\mathrm{sc}}$ and $\sigma_{\mathrm{abs}}$ are the scattering and absorption cross sections, respectively.

In view of the high linear transmission $(>90 \%$ when corrected for reflection losses), one can neglect $\sigma_{\text {abs }}$, so that $\sigma_{\text {ext }} \simeq \sigma_{\mathrm{sc}}$. Equation (4.1) is therefore

$$
\ln \left(T_{N}\right)=-\eta l \sigma_{\mathrm{sc}} .
$$

According to the Mie theory ${ }^{24}$ this scattering cross section depends on the scattering center radius and the wavelength: for spherical bubbles in a simple scattering regime, it is given by

$$
\sigma_{\mathrm{sc}}=\frac{2 \pi}{k^{2}} \sum_{n=1}^{\infty}(2 n+1)\left(\left|a_{n}\right|^{2}+\left|b_{n}\right|^{2}\right),
$$

where $k$ is the light wave vector, and $a_{n}$ and $b_{n}$ are the scattering coefficients, defined as

$$
\begin{aligned}
a_{n} & =\frac{m \Psi_{n}(m x) \Psi_{n}^{\prime}(x)-\Psi_{n}(x) \Psi_{n}^{\prime}(m x)}{m \Psi_{n}(m x) \xi_{n}^{\prime}(x)-\Psi_{n}^{\prime}(m x) \xi_{n}(x)} \\
b_{n} & =\frac{\Psi_{n}(m x) \Psi_{n}^{\prime}(x)-m \Psi_{n}(x) \Psi_{n}^{\prime}(m x)}{\Psi_{n}(m x) \xi_{n}^{\prime}(x)-m \Psi_{n}^{\prime}(m x) \xi_{n}(x)}
\end{aligned}
$$

$\Psi_{n}$ and $\xi_{n}$ are the Riccati-Bessel functions: $\Psi_{n}$ $=\rho J_{n}(\rho)$ and $\xi_{n}=\rho h_{n}^{(1)}(\rho)$, where $J_{n}(\rho)$ are the spherical Bessel functions, and $h_{n}^{(1)}(\rho)$ are the spherical Hankel functions of first order. $x$ and $m$ correspond to the size parameter and the relative refractive index, respectively, defined as functions of the refractive indices of the particle $(n)$ and of the surrounding medium $\left(n_{1}\right)$ and the sphere radius $(R)$ as

$$
\begin{gathered}
x=\frac{2 \pi n R}{\lambda}, \\
m=\frac{n_{1}}{n} .
\end{gathered}
$$

Therefore from the equations above, one sees that the wavelength and the particle radius determine the scattering cross section in a unique manner. On the other hand, the scattering cross section depends on the volume concentration $\eta$ through Eq. (4.3). One can therefore fit each nanosecond and picosecond experimental curve displayed in Section 3 (Fig. 3) with only two free parameters $R$ and $\eta$. This allows obtention of the evolution of average radius and concentration of the scattering centers in the medium. This procedure is of course not very precise, but it nevertheless gives a good order of magnitude of these parameters. This adjustment is more precise when the radii are small, on the condition that the scattered signal is strong enough to yield measurable effects.
Moreover, by fitting the transmittance curves at different delays, in the nanosecond regime (like those presented in Fig. 4), one determines the temporal evolution of the size and of the concentration of scattering centers, and we obtain the gas volume fraction (averaged on the probe light path), given by $V=(4 \pi / 3) \eta R^{3}$.

Figure 6 displays the volume fractions of the gas obtained from nanosecond pump-probe experimental results at $532 \mathrm{~nm}$ with SWNT and MWNT, both suspended in water. They increase rapidly and reach maxima of $1.4 \times 10^{-7}$ and $1.2 \times 10^{-7}$, and $7 \mathrm{~ns}$ and $10 \mathrm{~ns}$, after the top of the pump pulse for SWNT and MWNT, respectively. The decrease of the volume is much slower than the increase due to a slow cooling down of the medium after the pump pulse. We note that the (half-life) relaxation time is longer for SWNT ( $\tau \approx 30 \mathrm{~ns}$ ) than for MWNT ( $\tau \approx 15 \mathrm{~ns}$ ), but the reason for this is still unclear. These relaxation times are the same as those obtained for relaxation of the probe perturbation. As evidenced in Fig. 6, we have a good correlation between the time evolution of both the probe transmission and the gas volume fraction inferred from the multiwavelength probe-transmittance measurements.

Figure 7 shows the evolution of the concentration $\eta$ and average radius $R$ of scattering centers for SWNT in water. Surprisingly, the concentration of scattering centers decreases very rapidly, in the first few nanoseconds follow-

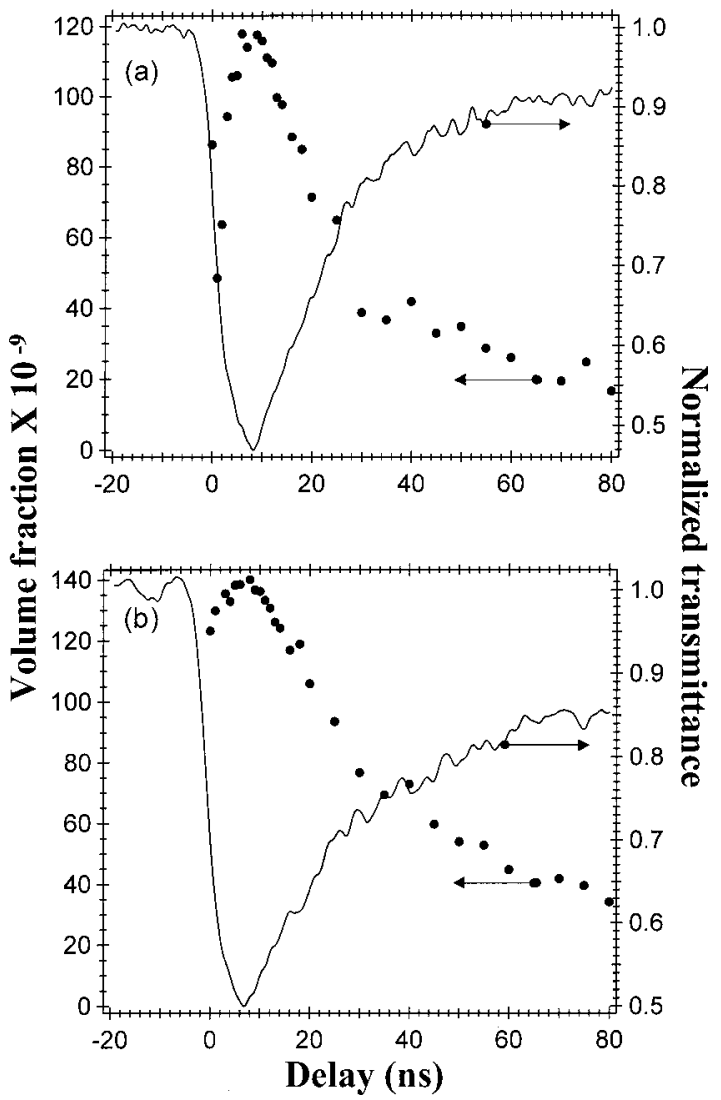

Fig. 6. Evolution of the gas volume fraction (closed circle) and normalized transmittance of the probe (solid curve) for (a) SWNT and for (b) MWNT suspended in water, in the nanosecond regime, at $532 \mathrm{~nm}$ and for input energy of $200 \mathrm{~mJ} / \mathrm{cm}^{2}$. Delay zero corresponds to the maximum of the pump pulse. The linear transmittance is $80 \%$ for all samples. 


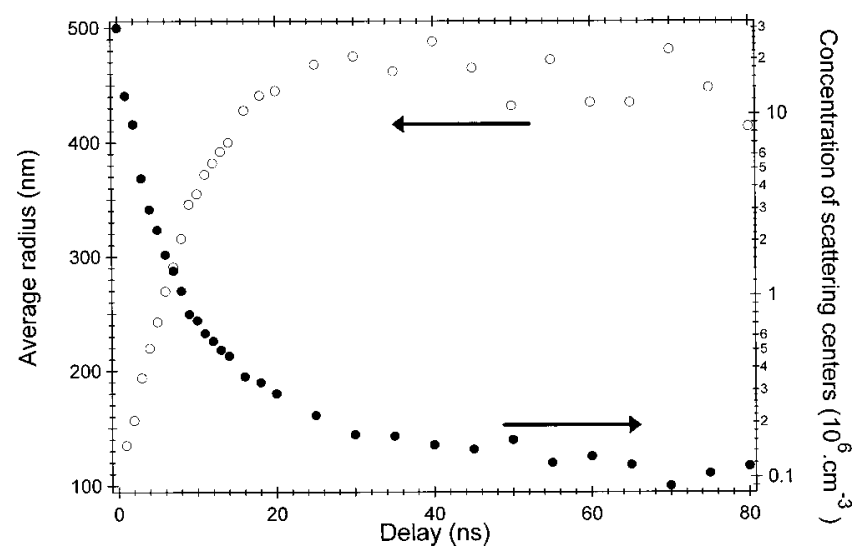

Fig. 7. Evolution of the concentration and of the average radius of scattering centers for SWNT in water, for 532-nm nanosecond pump pulses and for input energy of $200 \mathrm{~mJ} / \mathrm{cm}^{2}$. Delay zero corresponds to the maximum of the pump pulse. The linear transmittance is $80 \%$ for all samples.
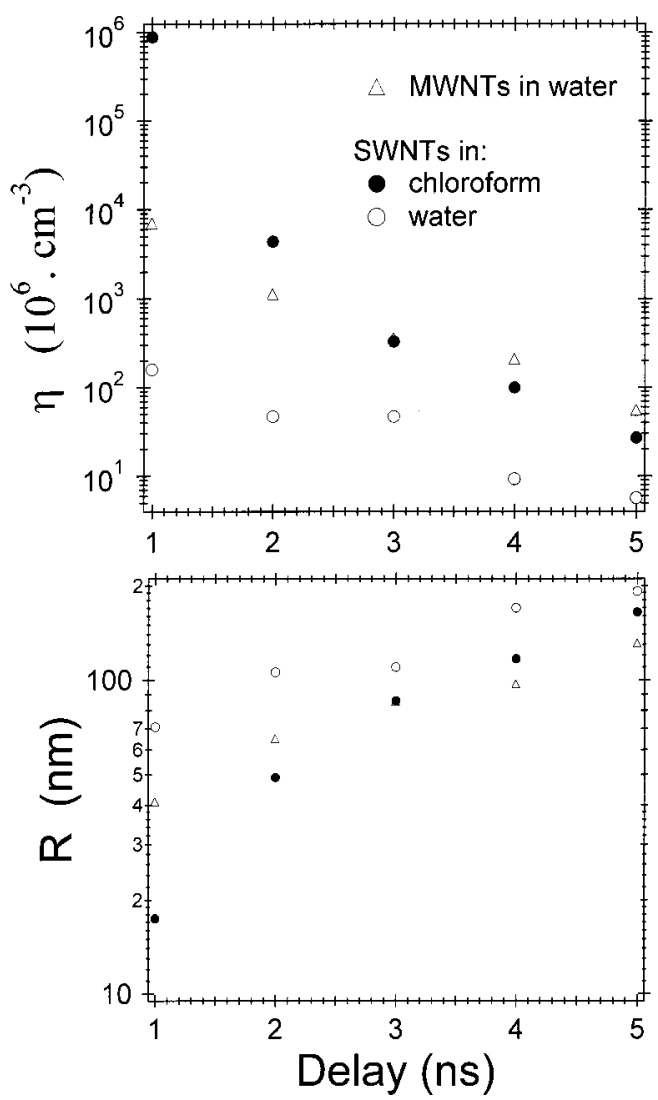

Fig. 8. Evolution of (a) the concentration and of (b) the average radius of scattering centers for SWNT and MWNT in water and SWNT in chloroform, for 532-nm picosecond pump pulses for input energy of $600 \mathrm{~mJ} / \mathrm{cm}^{2}$. Delay zero corresponds to the maximum of the pump pulse. The linear transmittance is $80 \%$ for all samples.

ing the top of the pump pulse. Symmetrically, the average radius increases and reaches a plateau after approximately $25 \mathrm{~ns}$. The decrease of the concentration is associated with an increase of the average radius because of a competition between coalescence and condensation of vapor bubbles.

With the results of the picosecond experiments, one can focus on the beginning of the bubble growth (Fig. 8). For
SWNT, the average scattering radius is lower in chloroform than in water suspensions, indicating that coalescence is more important in water suspensions, leading to larger vapor bubbles. However, the concentration of scattering centers is more important in chloroform than in water suspensions. These features show that the behaviors in the two solvents are quite different: the probe beam is scattered by a large number of small vapor bubbles in chloroform suspensions, whereas in water suspensions, it is scattered by a smaller number of larger vapor bubbles. This difference can be related to the higher surface tension at the liquid/gas interface in water than in chloroform: to minimize surface energy, aggregation and subsequent coalescence of vapor bubbles are faster in water than in chloroform, leading to the formation of larger scattering centers in the few nanoseconds following the pump pulse. With a pump wavelength of $1064 \mathrm{~nm}$, we obtained similar results.

\section{CONCLUSION}

We carried out picosecond and nanosecond pump-probe experiments with a variable probe wavelength. These experiments allow determination of the concentration and average radius of scattering centers as well as their volume fraction as a function of time, by a simple extinction model based on Mie theory. From that analysis, we show a clear correlation between the evolutions of the probe transmittance and of the gas volume fraction. In water suspensions, coalescence of vapor bubbles begins in the first nanosecond following the pump pulse. The coalescence effect is slower in chloroform suspension due to lower surface tension at the liquid/gas interfaces. In chloroform suspensions, the probe is scattered by many small vapor bubbles while in water suspensions, it is scattered by a smaller number of larger vapor bubbles. However, chloroform suspensions exhibit a better opticallimiting efficiency than water suspensions in the nanosecond regime. The pump-probe experiments also confirmed that carbon-nanotube suspensions are efficient only for nanosecond and longer pulses. The coalescence phenomenon was recently confirmed by shadowgraphic measurements, which will be published elsewhere.

\section{ACKNOWLEDGMENTS}

The authors greatly appreciate the high-quality samples from S. Tahir and P. Bernier. We thank M. Andrieux and F. Lafonta for their help and fruitful discussion. E. Anglaret acknowledges the Délégation Générale pour l'Armement for financial support.

\section{REFERENCES}

1. K. Nashimoto, R. Pachter, B. W. Wessels, J. Shmulovich, A. K.-Y. Jen, K. Lewis, R. Sutherland, and J. W. Perry, eds., Thin Films for Optical Waveguides Devices and Materials for Optical Limiting, Vol. 597 of MRS Proceedings Series (Materials Research Society, Warrendale, Pa., 1999).

2. R. Bozio, F. Kazjar, and M. Meneghetti, eds., Proceeding of Second International Workshop on Optical Power Limiting (Gordon and Breach, London, 2002).

3. J. W. Perry, "Organics and metal-containing reverse saturable absorbers for optical limiters," in Nonlinear Optics of 
Organics Molecules and Polymers, H. S. Nalwa and S. Miyata, eds. (CRC Press, Orlando, Fla., 1997), pp. 813-840.

4. P. Feneyrou, "Broadband optical limiting using tandem filters with multiphoton absorber and reverse saturable absorbers," J. Opt. Nonlinear Phys. Mat. 9, 523-530 (2000).

5. R. W. Boyd, Nonlinear Optics (Academic, New York, 1992).

6. B. L. Justus, A. L. Huston, and A. J. Campillo, "Broadband thermal optical limiter," Appl. Phys. Lett. 63, 1483-1486 (1993).

7. K. J. McEwan, P. K. Milsom, and D. B. James, "Nonlinear optical effects in carbon suspensions," Proc. SPIE 3472, 42-52 (1998).

8. K. M. Nashold and D. P. Walter, "Investigations of optical limiting mechanisms in carbon particle suspensions and fullerene solutions," J. Opt. Soc. Am. B 12, 1228-1237 (1995).

9. K. Mansour, M. J. Soileau, and E. W. Van Stryland, "Nonlinear optical properties of carbon-black suspensions (ink)," J. Opt. Soc. Am. B 9, 1100-1109 (1992).

10. V. Joudrier, P. Bourdon, F. Hache, and C. Flytzanis, "Characterization of nonlinear scattering in colloidal suspensions of silica particles," Appl. Phys. B 70, 105-109 (2000).

11. S. Iijima, "Helical microtubules of graphitic carbon," Nature 354, 56-58 (1991).

12. L. Vivien, E. Anglaret, D. Riehl, F. Bacou, C. Journet, C. Goze, M. Andrieux, M. Brunet, F. Lafonta, P. Bernier, and F. Hache, "Single-wall carbon nanotubes for optical limiting," Chem. Phys. Lett. 307, 317-319 (1999), and erratum 312 , 617 (1999).

13. S. R. Mishra, H. S. Rawat, S. C. Mehendale, K. C. Rustagi, A. K. Sood, R. Bandyopadhyay, A. Govindaraj, and C. N. R. Rao, "Optical limiting in single-walled carbon nanotube suspensions," Chem. Phys. Lett. 317, 510-514 (2000).

14. L. Vivien, E. Anglaret, D. Riehl, F. Hache, F. Bacou, M. Andrieux, F. Lafonta, C. Journet, C. Goze, M. Brunet, and P.
Bernier, "Optical limiting properties of singlewall carbon nanotubes," Opt. Commun. 174, 271-275 (2000).

15. X. Sun, Y. Xiong, P. Chen, J. Lin, W. Ji, J. Hong Lim, S. S. Yang, D. J. Hagan, and E. W. Van Stryland, "Investigation of an optical limiting mechanism in multiwalled carbon nanotubes," Appl. Opt. 39, 1998-2001 (2000).

16. L. Vivien, D. Riehl, E. Anglaret, and F. Hache, "Pump-probe experiments at $1064 \mathrm{~nm}$ in singlewall carbon nanotube suspensions," IEEE J. Quantum Electron. 36, 680-686 (2000).

17. L. Vivien, D. Riehl, F. Hache, and E. Anglaret, "Nonlinear scattering origin in carbon nanotube suspensions," J. Opt. Nonlinear Phys. Mat. 9, 297-308 (2000).

18. L. Vivien, D. Riehl, P. Lançon, F. Hache, and E. Anglaret, "Pulse duration and wavelength effects on the optical limiting behavior of carbon nanotube suspensions," Opt. Lett. 26, 223-225 (2001).

19. J. E. Riggs, D. B. Walker, D. L. Carroll, and Y.-P. Sun, "Optical limiting properties of suspended and solubilized carbon nanotubes," J. Phys. Chem. B 104, 7071-7076 (2000).

20. C. Journet, W. K. Maser, P. Bernier, A. Loiseau, M. Lamy de la Chapelle, S. Lefrant, P. Deniard, R. Lee, and J. E. Fischer, "Large-scale production of single-walled carbon nanotubes by the electric-arc technique," Nature 388, 756-758 (1997).

21. L. Vaccarini, C. Goze, R. Aznar, V. Micholet, C. Journet, and P. Bernier, "Purification procedure of carbon nanotubes," Synth. Metals 103, 2492-2493 (1999).

22. S. Rols, E. Anglaret, J. L. Sauvajol, G. Coddens, and A. J. Dianoux, "Neutron scattering studies of the structure and dynamics of nanobundles of single wall carbon nanotubes," Appl. Phys. A 69, 1-72 (1999).

23. S. Rols, R. Almairac, L. Henrard, E. Anglaret, and J. L. Sauvajol, "Diffraction by finite-size crystalline bundles of single wall nanotubes," Europhys. J. B 10, 263-270 (1999).

24. C. F. Bohren and D. R. Huffman, Absorption and Scattering of Light by Small Particles (Wiley, New York, 1983). 